\title{
Nanorheometry of Molecularly Thin Liquid Lubricant Films Coated on Magnetic Disks
}

\author{
Shintaro Itoh, ${ }^{1}$ Yuya Hamamoto, ${ }^{1}$ Koki Ishii, ${ }^{1}$ Kenji Fukuzawa, ${ }^{1}$ and Hedong Zhang ${ }^{2}$ \\ ${ }^{1}$ Department of Micro-Nano Systems Engineering, Nagoya University, Furo-cho, Chikusa-ku, Nagoya, Aichi 464-8603, Japan \\ ${ }^{2}$ Department of Complex Systems Science, Nagoya University, Furo-cho, Chikusa-ku, Nagoya, Aichi 464-8601, Japan \\ Correspondence should be addressed to Shintaro Itoh, s_itoh@nuem.nagoya-u.ac.jp
}

Received 3 August 2012; Revised 20 November 2012; Accepted 4 December 2012

Academic Editor: Tom Karis

Copyright ( $) 2012$ Shintaro Itoh et al. This is an open access article distributed under the Creative Commons Attribution License, which permits unrestricted use, distribution, and reproduction in any medium, provided the original work is properly cited.

\begin{abstract}
Molecularly thin lubricant films are used for the lubrication of head disk interfaces in hard disk drives. The film thickness is reduced to 1-2 nm to minimize the magnetic spacing, and optimal, precise design is required to obtain sufficient lubrication. However, until now, there was no generally applicable method for investigating such thin films. Therefore, we developed a highly sensitive shear force measuring method and have applied it to the viscoelastic measurement of lubricant films coated on magnetic disk surfaces. In this paper, we review the method and summarize the useful findings we have demonstrated so far.
\end{abstract}

\section{Introduction}

High-recoding-density hard disk drives (HDDs) require the magnetic spacing to be as small as possible. Lubrication of the head disk interface (HDI) is achieved by a diamondlike carbon (DLC) overcoat on the magnetic layer and a liquid lubricant film on the DLC surface. Thus, the thicknesses of both these thin films must be minimized to reduce the magnetic spacing. Liquid lubricant films are typically $1-2 \mathrm{~nm}$ thick, which corresponds to a monolayer thickness. In addition, the magnetic head comes closer to the surface of liquid lubricant film and recently its gap was around $1 \mathrm{~nm}$. A further increase in the recording density of HDDs will require the head to be in contact with the lubricant film surface. Although air bearing still plays an important role in maintaining the flying height, the lubrication regime between the head and disk changes from hydrodynamic to boundary lubrication. To guarantee HDD performance, effective design of the lubricant film becomes a crucial requirement. However, until recently there was no generally applicable method for characterizing the mechanical properties of such a thin film. A surface force apparatus (SFA) is a pioneering method that can measure the viscoelastic properties of a molecularly thin liquid film confined in two mica surfaces under shearing motion, and experiments using the SFA have revealed the thin films have unique mechanical properties different from those in the bulk state, such as an enhanced viscosity and prolonged retardation time [1-3]. Understanding physical properties of molecularly thin liquid films is one of the important subjects not only in the HDI tribology but also in the field of soft condensed matter. Such a thin film is considered as one type of confinement. The confinement has various types such as liquids in porous media, and water in a cell, polymer blends, and so on [4]. The measurement methods typically used are dielectric spectroscopy, neutron scattering, NMR, differential scanning calorimetry, ellipsometry, and so on [4-7]. Jackson and McKenna observed large decrease of glass transition temperature $T_{\mathrm{g}}$ with liquids confined in nanometer size pores by using colorimetric measurements $[8,9]$. This result means the mobility of confined liquids can increase. Similar results were obtained not only with liquids in pores but also with thin liquid films [10-13]. The thin films include free standing films and those coated on substrates. However, to the contrary, increase of $T_{\mathrm{g}}$ or no effect on $T_{\mathrm{g}}$ were also observed with similar confined systems [14-19]. These controversial and contradictory results are due to complexity of the phenomena and wide variety of the measurement methods and the universal explanation has not been made. He et al. described through solvation dynamics experiments that whether $T_{\mathrm{g}}$ increase or decrease depends 
on the condition of confining surface, which is "soft" or "hard" [20]. In the case of soft surface, $T_{\mathrm{g}}$ decreased. The hard surface suppressed the molecular mobility and it led to the $T_{\mathrm{g}}$ increase. Based on the experimental results obtained by using the fluorescence probes, Torkelson's group reported that thin films coated on substrates had $T_{\mathrm{g}}$ gradient from the free surface to the substrate [21]. The molecular mobility decreased as the substrate was approached, which led to the $T_{\mathrm{g}}$ increase. In the case of liquid lubricant films used in HDI, they are confined in two solid substrates during shearing. The same type of confinement was realized in the SFA and the experimental results indicated the decrease of molecular mobility, which corresponds to the $T_{\mathrm{g}}$ increase. However, the solid substrates that confine the liquid film are limited to mica in the SFA measurements. In addition, dynamic measurements are limited to frequencies below $100 \mathrm{~Hz}$. Many of the experimental results in previous studies show that the lubrication characteristics of the magnetic disk surface depend largely on the interaction between the DLC surface and the lubricant molecules $[22,23]$. Therefore, the liquid lubricant films used for the HDI lubrication must be characterized on the DLC surface. Additionally, since the relative speeds between the head and disk are quite high, highly dynamic measurements are preferred. To measure the dynamic viscoelastic properties of molecularly thin lubricant films on the magnetic disk surface, we developed a highly sensitive shear force measuring method. We call the method the fiber wobbling method (FWM) [24]. In this paper, we review our previous research concerning the FWM and introduce our major results including some new findings.

\section{Fiber Wobbling Method}

2.1. Concept. There are two major requirements for the viscoelastic measurements of molecularly thin liquid films under shearing motion. One is the sensitive detection of the shear force and the other is the precise control of the shearing gap width. The FWM concept meets both of these demands. We use an optical fiber with a spherical end as the shearing probe and set it to be perpendicular to the sample surface. During the shearing of the sample by the probe tip, the shear force acting on the tip can be measured by detecting the fiber deflection as shown schematically in Figure 1(a). Since the rigidity of the probe in the axial direction is much higher than that in the bending direction, we can attain both a high shear force sensitivity and precise gap control.

In the dynamic viscoelastic measurements using the FWM, the probe was oscillated sinusoidally at a constant frequency and amplitude. The amplitude change and phase shift of the probe tip oscillation was measured, and these values were used to obtain the viscoelastic properties of the lubricant films. Since the size of the probe is typically a few millimeters in length and $100 \mu \mathrm{m}$ in diameter (Figure $1(\mathrm{~b})$ ), the natural frequency of the probe is higher than a few kilohertz. This means that dynamic measurements with frequencies of up to a few kilohertz can be achieved with the FWM. The spherical end of probe was made by laser melting and its root-mean-square roughness is typically around $0.2 \mathrm{~nm}$.

2.2. Shear Force Measurement. The shear force $F$ acting on the probe tip can be estimated using the following equation [2]:

$$
F=\eta \Omega U
$$

where $\eta$ is the viscosity of the lubricant and $U$ is the sliding speed. The parameter $\Omega$ is a geometrical parameter determined from the shape and gap of the two sliding surfaces. When a sphere of diameter $d$, which represents the probe tip, moves parallel to the plane surface with a gap $h, \Omega$ is written as:

$$
\Omega=3 \pi d\left[\frac{8}{15} \log \left(\frac{d}{h}\right)+\cdots\right] \approx \frac{8}{5} \pi d \log \left(\frac{d}{h}\right) .
$$

The approximation holds when $h \ll d$. Substituting typical values of $\eta=0.1 \mathrm{~Pa} \cdot s, U=10 \mathrm{~mm} / \mathrm{s}, h=1 \mathrm{~nm}$, and $d=200 \mu \mathrm{m}$ into (1) and (2) gives an estimate of $F$ to be on the order of $1 \mathrm{nN}$. Since the spring constant of the probe is around $10 \mathrm{~N} / \mathrm{m}$, we have to attain a sensitivity of $0.1 \mathrm{~nm}$ in the displacement measurement of the probe deflection. To meet this requirement, we developed an original method as shown in Figure 2. We use the fiber as a lens to focus the laser onto a position sensitive detector (PSD). The laser beam is incident in a direction perpendicular to the plane of probe deflection. When the fiber is deflected, the laser spot on the PSD moves, and therefore, the amount of deflection can be obtained from the displacement of the laser spot. The sensitivity of the measurement strongly depends on the numerical aperture of the objective lens and the distance between the lens and probe. The optimal conditions were determined by a numerical calculation using a ray tracing method and experimentally verified. The details of the optical system design are described in [25]. Figure 3 shows the experimentally obtained relationship between the probe amplitude and signal-to-noise ratio. In the experiment, the probe was oscillated sinusoidally with the amplitude synchronously detected by a lock-in amplifier. The data represented by the circles, triangles, and squares correspond to numerical apertures of $0.3,0.25$, and 0.1 , respectively. A least-squares method is used to fit the experimental data, and the results are shown as the straight lines. From the fitted functions, we determine the detection limit of the measurement to be the probe amplitude for a signal-tonoise ratio of 1 . Consequently, the detection limits are 0.006 , 0.007 , and $0.01 \mathrm{~nm}$ for $N=0.3,0.25$, and 0.1 , respectively. This corresponds to a shear force sensitivity on the order of $0.1 \mathrm{nN}$, and we can thus conclude that the sensitivity of the FWM is sufficient for the measurement of molecularly thin films.

2.3. Precise Control and Determination of the Gap Width. The width of the shearing gap, which is the width of the gap between the probe tip and substrate, is controlled by a piezo stage on which the sample is mounted. Since the longitudinal rigidity of the probe is sufficiently high, the 


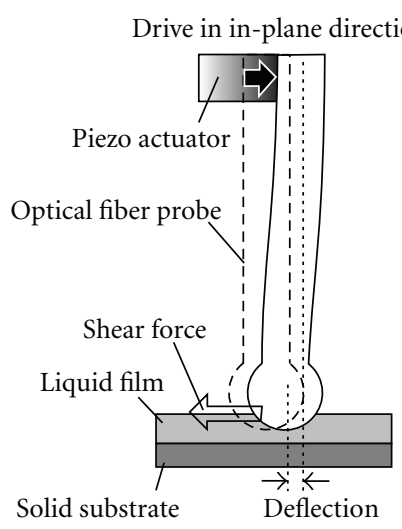

(a)

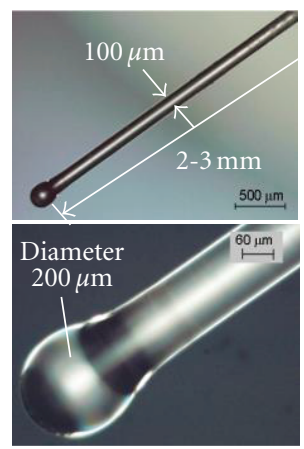

(b)

FIgURE 1: (a) Concept of shear force detection in the fiber wobbling method and (b) micrographs of the optical fiber probe.

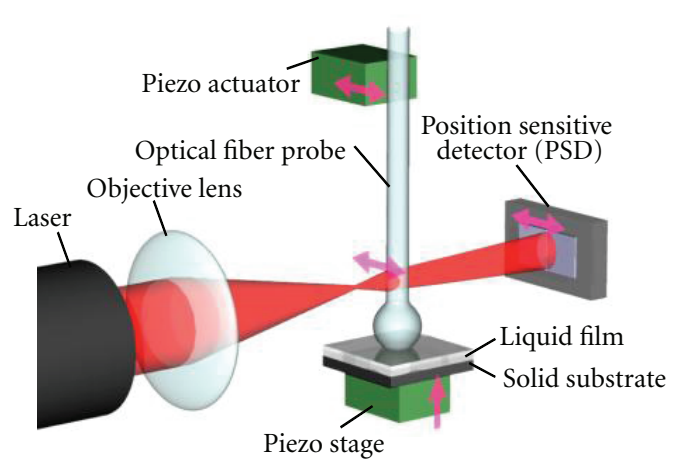

FIGURE 2: Schematic diagram of the fiber wobbling method (FWM).

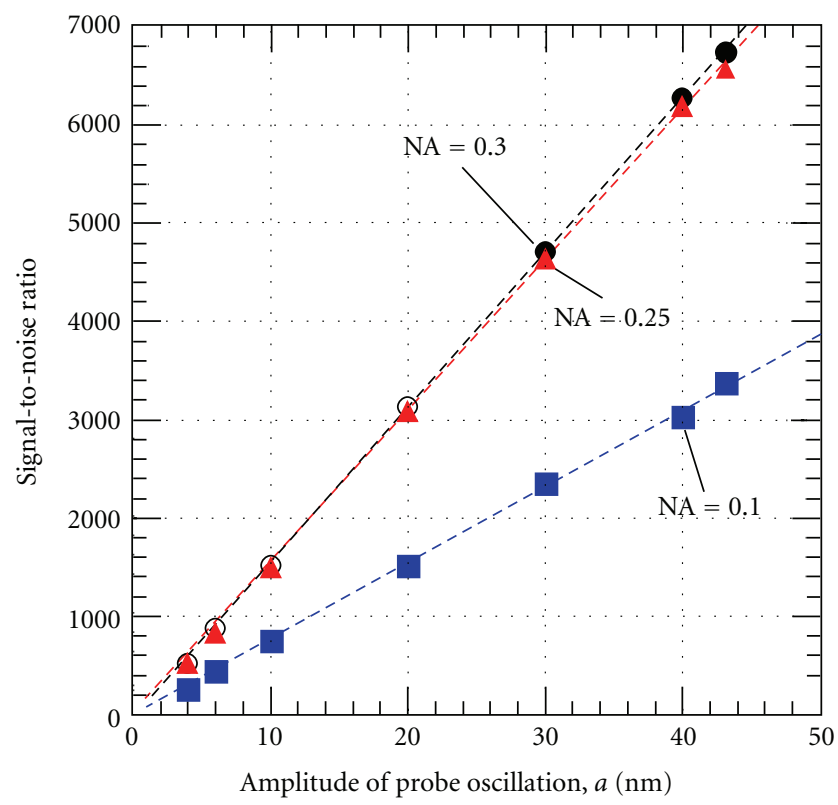

FIGURE 3: Experimental results of the measured relationship between the probe oscillation amplitude and the signal-to-noise ratio of the displacement measurement. change in the gap width is equal to the displacement of the piezo stage. Therefore, we can control the gap width with the same resolution as that of the piezo stage, which is $0.1 \mathrm{~nm}$. However, the absolute value of the gap width cannot be measured directly. Therefore, we determine the solid contact point experimentally, define it as the zero point of the gap width, and the shearing gap is determined by the displacement of the piezo stage from this point. Thus, the probe tip must make contact with the solid substrate at least once in the measurements. The basic experimental procedure in the FWM measurements is shown schematically in Figure 4. In the initial state, the gap was set to be much larger than a nanometer-size gap, such as $1 \mu \mathrm{m}$, and the sinusoidal oscillation of the probe at a constant frequency and amplitude was started. The gap was then gradually decreased at a constant rate, which is typically a few nanometers per second. As the gap width decreased, we synchronously measured the amplitude and phase of the probe tip oscillation, detected by the optical method described in Section 2.2, using the lock-in amplifier. The measurement was stopped when solid contact occurred.

According to the above procedure, the determination of the gap width depends on the sensitivity of the solid contact point detection. For this purpose, we developed an original technique that detects the excitation of the resonant oscillation of the shearing probe [26]. In the initial stage of solid contact, intermittent asperity contact occurs between two sliding surfaces owing to the surface roughness. The asperity contact causes an impulsive force to act on the probe tip and excites the resonant oscillation of the probe. We detect solid contact by monitoring the increase in the resonant oscillation amplitude. The frequency of the forced oscillation during lubricant shearing is much smaller than the natural frequency of the probe, and so two lock-in amplifiers can be used to measure the forced oscillation component and the resonant oscillation component separately in different frequency ranges. This technique enables us to define the solid contact point with an accuracy on the order of $0.1 \mathrm{~nm}$. 


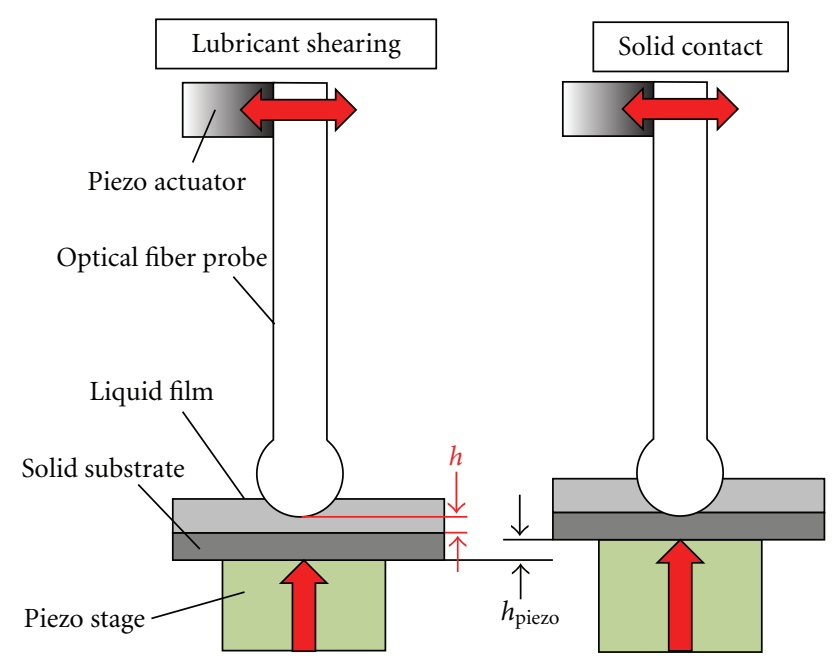

FIGURE 4: Gap determination procedure in the FWM measurements. Shearing gap $h$ is equal to the displacement of the piezo stage until the solid contact occurs, which is represented by $h_{\text {piezo }}$.

TABLE 1: Lubricants used in the experiment.

\begin{tabular}{lccc}
\hline & $\mathrm{Z} 03$ & $\mathrm{Zdol} 2000$ & $\mathrm{Zdol} 4000$ \\
\hline Main chain structure & $\mathrm{X}-\mathrm{CF}_{2}\left(\mathrm{OCF}_{2} \mathrm{CF}_{2}\right)_{m}\left(\mathrm{OCF}_{2}\right)_{n} \mathrm{OCF}_{2}-\mathrm{X}$ \\
End groups (-X) & $-\mathrm{CF}_{3}$ & $-\mathrm{CH}_{2} \mathrm{OH}$ & $-\mathrm{CH}_{2} \mathrm{OH}$ \\
Molecular weight (amu) & 4000 & 2000 & 4000 \\
Bulk Viscosity at RT (Pas) & 0.05 & 0.15 & 0.18 \\
\hline
\end{tabular}

\section{Viscoelastic Measurement with the Immersed System}

The probe tip was immersed into a relatively thick lubricant film (e.g., $200 \mu \mathrm{m}$ ), and the gap was decreased to the nanometer scale $[24,27]$. The lubricant film was confined in the nanometer-sized gap and its viscoelasticity was measured. In this immersed system, the gap between the two sliding surfaces is completely filled with the lubricant, and since the gap width varies with the radius of curvature of the probe tip, the obtained viscoelastic properties represent those of the average. However, since the shear force generated for the narrowest gap plays a dominant role in the measured mechanical response, we observe a strong dependency of the viscoelasticity on the gap variance when the lubricant is confined to the nanometer-sized gap. In immersed-system measurements, we can investigate the entire viscoelasticity transition process of confined lubricants as the gap changes from the micrometer to nanometer scale.

3.1. Materials and Method. We used a magnetic disk as the solid substrate, and the top layer of the disk is a DLC overcoat with a surface roughness of $0.59 \mathrm{~nm}$. As the lubricant, we used three different types of perfluoropolyether (PFPE) lubricants as listed in Table 1. All have the same main chain structure but the end groups differ: Zdol2000 and Zdol4000 (with different molecular weights) have hydroxyl end groups on both sides of the main chain, making them polar lubricants, and both ends of the Z03 main chain are terminated by fluorine, which means that Z03 is a nonpolar lubricant. The difference in polarity affects the affinity to the magnetic disk surface. The polar end groups are strongly adsorbed onto the DLC surface and chemical bonding can be formed. Lubricant molecules that are chemically adsorbed on the disk surface are known as immobile molecules or bonded molecules and those that are physically adsorbed are known as mobile molecules. The number of bonded molecules can be controlled by annealing or UV treatment.

We placed one drop of the lubricant on the disk surface and waited until the lubricant spread spontaneously over the surface and stabilized. The amount of liquid in the drop was controlled to produce a wet film with a thickness of around $200 \mu \mathrm{m}$. As mentioned in the previous section, since the mechanical response obtained during the shearing of lubricant is dominated by the lubricant confined in the narrowest gap, any variation in the immersion depth of the probe will have a negligible effect on the measurements. The sample was not annealed or UV treated.

A schematic diagram of the experimental setup is shown in Figure 5. The piezo actuator was used to oscillate the probe, and the gap between the probe tip and sample was controlled by the piezo stage. The deflection signal from the PSD, a current signal, was converted to a voltage and amplified by the $I-V$ converter. The amplified signal was divided into two lines; one line was detected by the lock-in amplifier that used the piezo actuator oscillation signal as a reference signal and its amplitude and phase were measured. The other line was detected by another lock-in amplifier to measure the amplitude of the resonant oscillation component for the solid contact point detection. The reference signal was generated by the function generator with a frequency equal to the natural frequency of the probe. These three measured values were simultaneously recorded on the computer via an analog-to-digital converter.

After the probe tip was immersed in the lubricant film and the shearing gap was set to around $1 \mu \mathrm{m}$, we started the probe oscillating at a frequency of $800 \mathrm{~Hz}$ and a peak-to-peak amplitude of $30 \mathrm{~nm}$. The gap was decreased at a constant rate of $10 \mathrm{~nm} / \mathrm{s}$, and the amplitude and phase of the probe oscillation was measured until solid contact occurred.

3.2. Calculation of Viscoelasticity. The mechanical model used to obtain the viscoelastic properties is shown in Figure 6. The equation of motion is expressed as

$$
m \frac{d^{2} x}{d t^{2}}+c \frac{d x}{d t}+k x=F_{0} e^{i \omega t}
$$

where $F_{0}, \omega, x, m, k$, and $c$ are the driving force, driving frequency, displacement, effective mass of the spherical tip, spring constant of the probe, and damping coefficient of the liquid lubricant, respectively. The solution for (3) is written as

$$
x=a \cos (\omega t-\delta)
$$




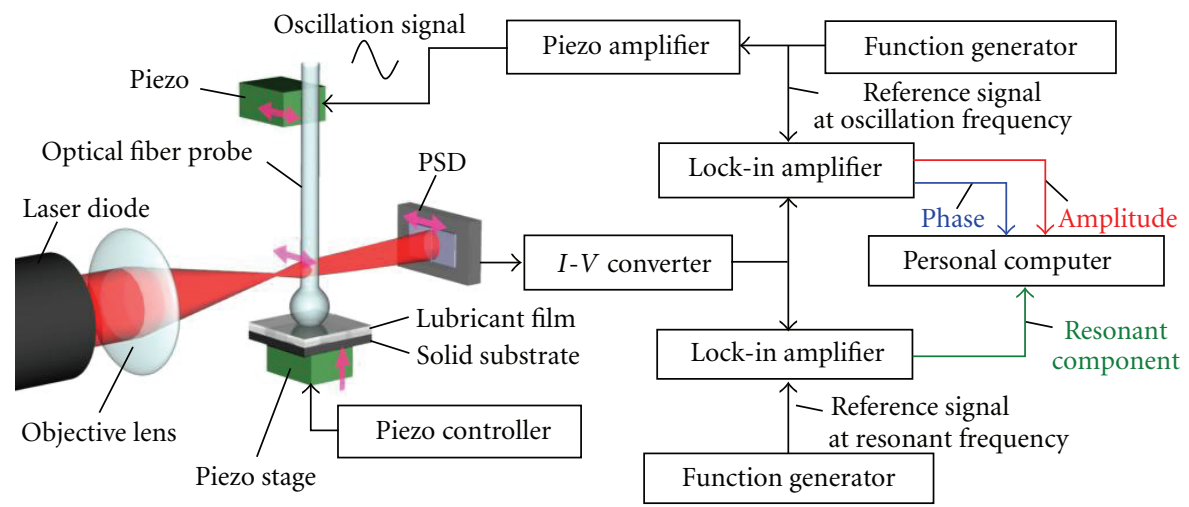

Figure 5: Experimental setup of the fiber wobbling method.

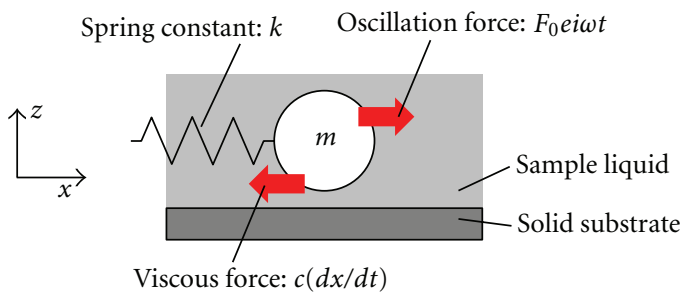

Figure 6: Mechanical model for the viscoelastic calculation.

where $a$ and $\delta$ are the amplitude and phase shift, respectively, defined as

$$
\begin{gathered}
a=\frac{F_{0}}{\left[\left(k-m \omega^{2}\right)^{2}+\omega^{2} c^{2}\right]^{1 / 2}}, \\
\delta=\tan ^{-1}\left(\frac{\omega c}{k-m \omega^{2}}\right) .
\end{gathered}
$$

The driving force $F_{0}$ is obtained by substituting $c=0$ and $a=a_{0}$ into (5), where $a_{0}$ represents the forced oscillation amplitude. The relationship between the damping coefficient and viscosity of the lubricant is expressed as follows:

$$
c=\eta_{\text {eff }} \Omega \text {. }
$$

Here, $\eta_{\text {eff }}$ represents the effective viscosity since lubricant confined in a nanometer-sized gap is expected to be nonNewtonian. In the immersed system, the sliding surfaces were assumed to be a sphere and plane with the sphere moving parallel to the plane surface. In this case, the geometric parameter $\Omega$ is given by (2). In our discussion on viscoelastic properties, the effective viscosity $\eta_{\text {eff }}$ is represented by a complex function:

$$
\eta_{\mathrm{eff}}=\eta^{\prime}-i \eta^{\prime \prime}
$$

where $\eta^{\prime}$ and $\eta^{\prime \prime}$ represent the viscosity and elasticity, respectively. From (3) to (8), we obtain $\eta^{\prime}$ and $\eta^{\prime \prime}$ as follows:

$$
\begin{gathered}
\eta^{\prime}=\frac{a_{0}\left(k-m \omega^{2}\right) \sin \delta}{a \omega \Omega}, \\
\eta^{\prime \prime}=\frac{\left(a_{0} \cos \delta-a\right)\left(k-m \omega^{2}\right)}{a \omega \Omega} .
\end{gathered}
$$

In these equations, the amplitude $a$ and phase shift $\delta$ are the measured values of the probe tip oscillation. The other parameters are known values from the experimental conditions and probe specifications.

3.3. Results and Discussion. Using (9), we calculated $\eta^{\prime}$ and $\eta^{\prime \prime}$ of each lubricant. The results are shown in Figures 7, 8, and 9 . The closed circles are the viscosity values and the open circles are the elasticity values. In all the samples, the viscosity rapidly increased as the gap was decreased to the nanometer scale. The maximum value of the viscosity was approximately several dozen times larger than the bulk-state viscosity (listed in Table 1). This is caused by the lack of molecular mobility in the nanometer-sized gap width.

In the bulk state, the Z03, Zdol2000, and Zdol4000 lubricants have no elastic properties. However, elasticity appeared for gaps of less than a few nanometers. In other words, the transition from a viscous liquid to a viscoelastic liquid occurred. The elasticity also increased monotonically as the gap decreased. In a narrow gap, the physically and chemically adsorbed molecules on both sides of the confining surfaces can interact with each other, and it is considered that the mobility of these adsorbed molecules was extremely suppressed and thus the lubricants exhibited elasticity. The elasticity appeared at different gap widths for each tested lubricant: widths of $6.5,13.2$, and $13.4 \mathrm{~nm}$ for Z03, Zdol2000, and Zdol4000, respectively. These differences are considered to be due to the higher affinity of the polar lubricants to the magnetic disk surface; the polar lubricants may have formed a thicker adsorption layer that led to the appearance of elasticity at a wider gap width.

\section{Viscoelastic Measurement with the Thin-Film System}

The FWM measurements were applied to thin lubricant films coated on magnetic disks as in the actual HDI [26, 28]. From the measured mechanical responses, we firstly discuss the deformation of the lubricant film caused by the approaching and separating probe tip, which imitates the touchdown and takeoff behavior in the HDI. Secondly, we discuss the effect 


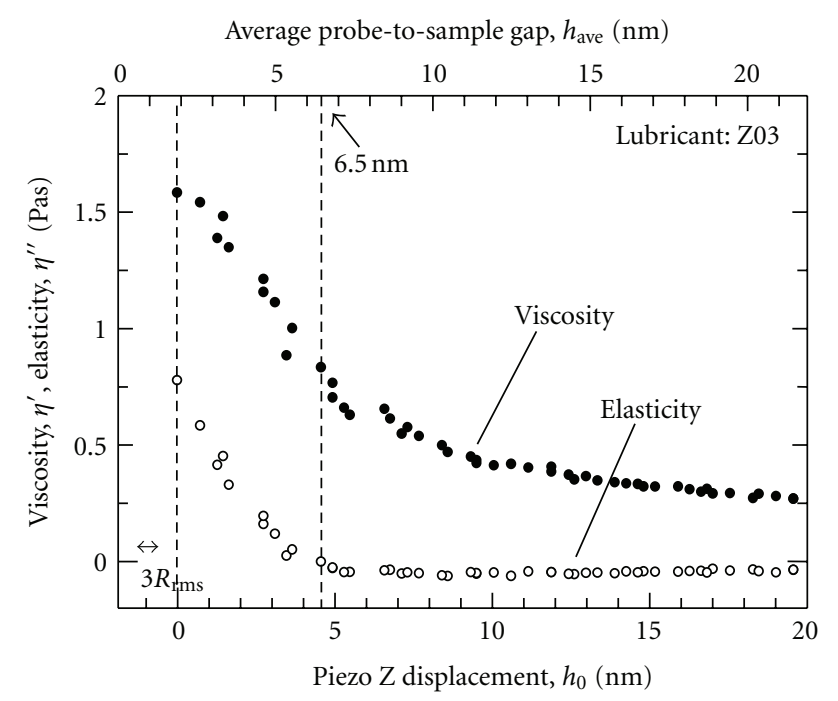

FIGURE 7: Effect of the shearing gap on the viscosity $\eta^{\prime}$ and elasticity $\eta^{\prime \prime}$ measured with the Z03 lubricant [24].

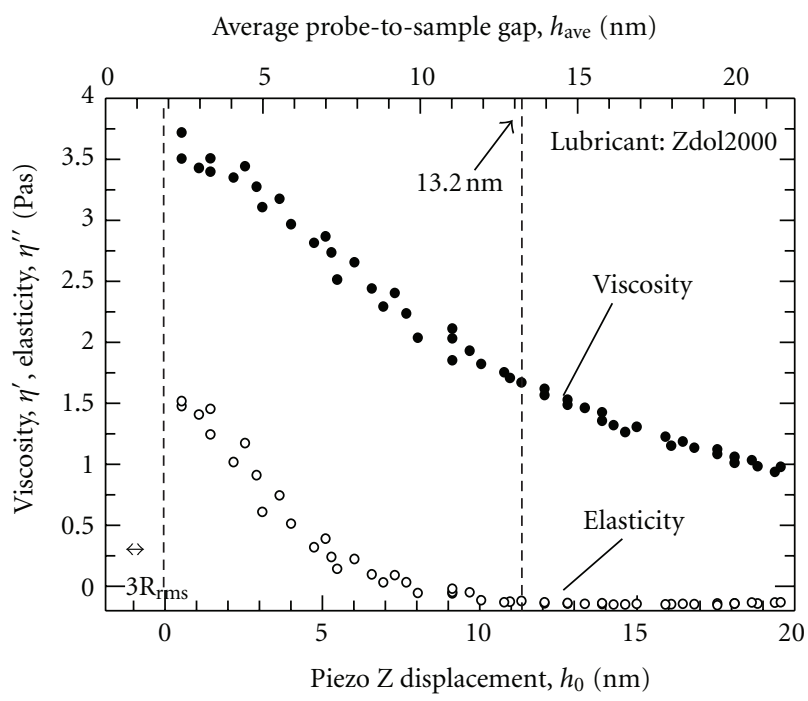

FIGURE 8: Effect of the shearing gap on the viscosity $\eta^{\prime}$ and elasticity $\eta^{\prime \prime}$ measured with the Zdol2000 lubricant [24].

of the bonding ratio on the viscoelasticity. Finally, the shear rate dependence of the viscosity is shown.

4.1. Materials and Method. The Z03 and Zdol4000 lubricants, specified in Table 1, and Ztetraol were coated onto a magnetic disk to form the samples. Ztetraol has the same main chain structure as Z03 and Zdol4000, though it has different end groups that are $\mathrm{X}=-\mathrm{CH}(-\mathrm{OH}) \mathrm{CH}_{2} \mathrm{OH}$. Since Ztetraol has two hydroxyl groups on each end of the main chain, it has higher affinity to the magnetic disk surface than Zdol4000. The lubricant films were formed by the dip-coating method. The disk coated with Zdol4000 was annealed, and samples that had different numbers of bonded molecules were prepared. The proportion of bonded

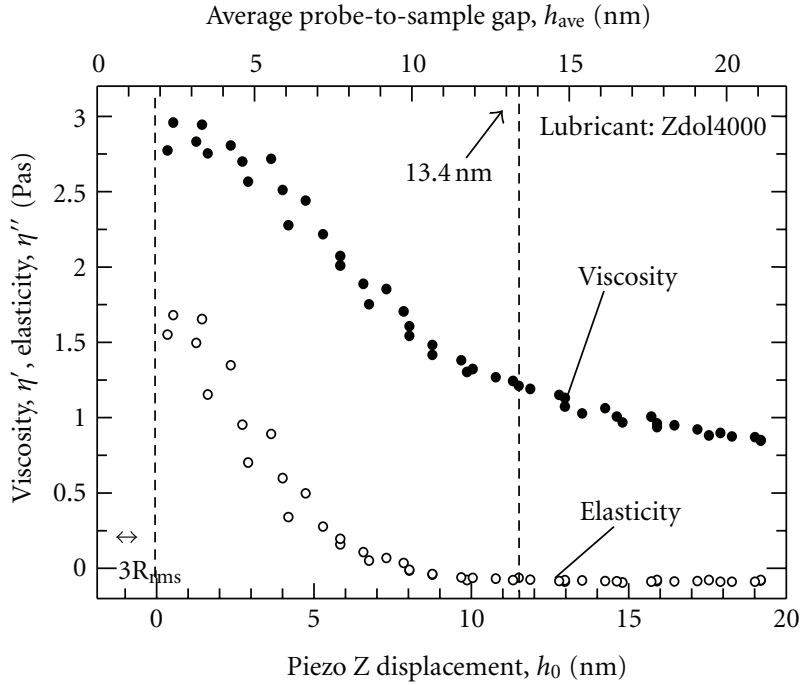

FIGURE 9: Effect of the shearing gap on the viscosity $\eta^{\prime}$ and elasticity $\eta^{\prime \prime}$ measured with the Zdol4000 lubricant [24].

TABLE 2: Samples prepared for the FWM measurements with the thin-film system.

\begin{tabular}{lcc}
\hline Type of lubricant & Film thickness, $h(\mathrm{~nm})$ & Bonding ratio, $b$ \\
\hline Z03 & 2.2 & 0 \\
Zdol4000 & 2.0 & 0.2 \\
Zdol4000 & 2.0 & 0.5 \\
Zdol4000 & 1.8 & 1.0 \\
Ztetraol & 1.9 & 0.3 \\
\hline
\end{tabular}

molecules is represented by the bonding ratio $b$, which is defined by

$$
b=\frac{h_{b}}{h} .
$$

Here, $h$ is the film thickness and $h_{b}$ is the film thickness measured after the sample has been rinsed with solvent, which means $h_{b}$ is the thickness composed only of the remaining bonded molecules. The bonding ratio was measured after all the viscoelastic measurements were finished, and an ellipsometer was used to measure the film thickness. The samples are listed in Table 2; since Z03 does not form chemically bonded molecules, its bonding ratio is 0 .

The experimental procedure was similar to that of the immersed-system measurement; in this case, the amplitude change and phase shift of the probe tip oscillation were measured with decreasing gap rate of $3 \mathrm{~nm} / \mathrm{s}$. In the thin-film system, the probe does not make contact with the sample at the beginning of the measurement since the initial gap is set to be much larger than the film thickness. Therefore, the measured mechanical response is divided into three sequential stages: noncontact, lubricant shearing, and solid contact. We also measured the mechanical response as the gap was increased at a rate of $3 \mathrm{~nm} / \mathrm{s}$ from the solid contact point. We call the former procedure the approaching process and the latter the separating process. The separating process 


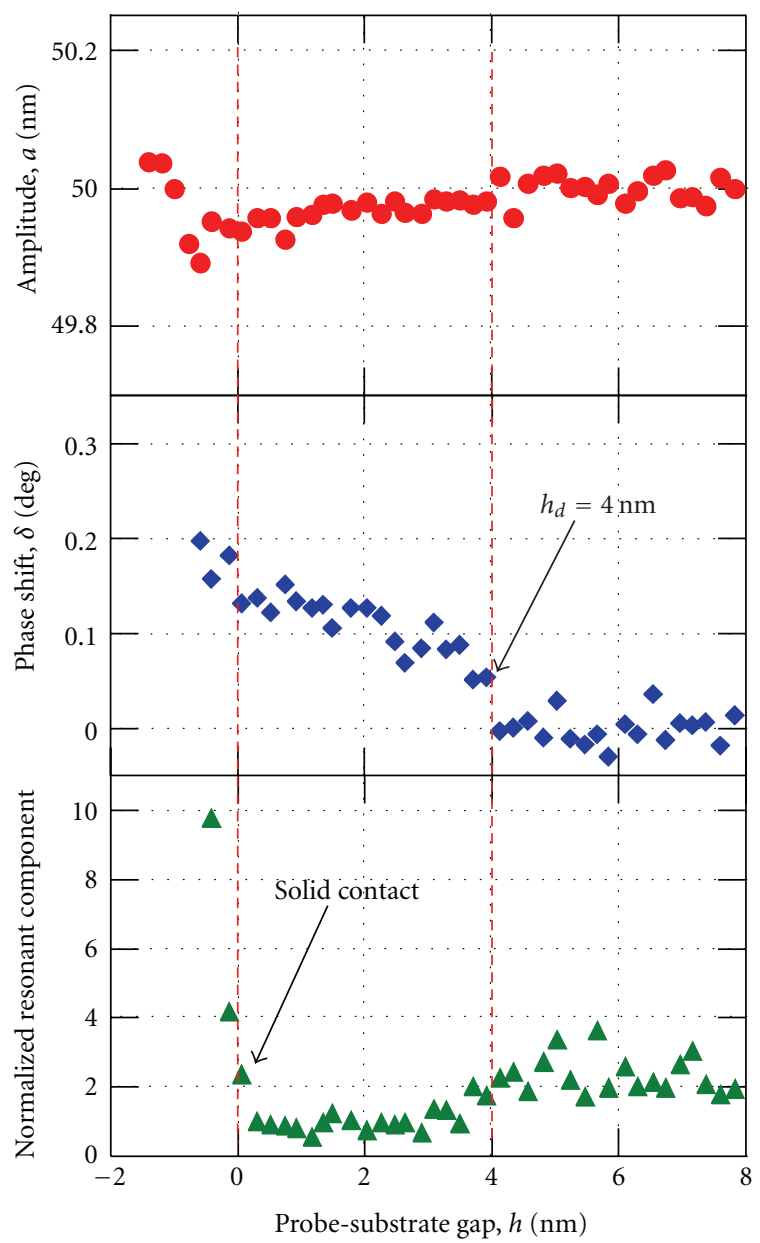

(a) Z03

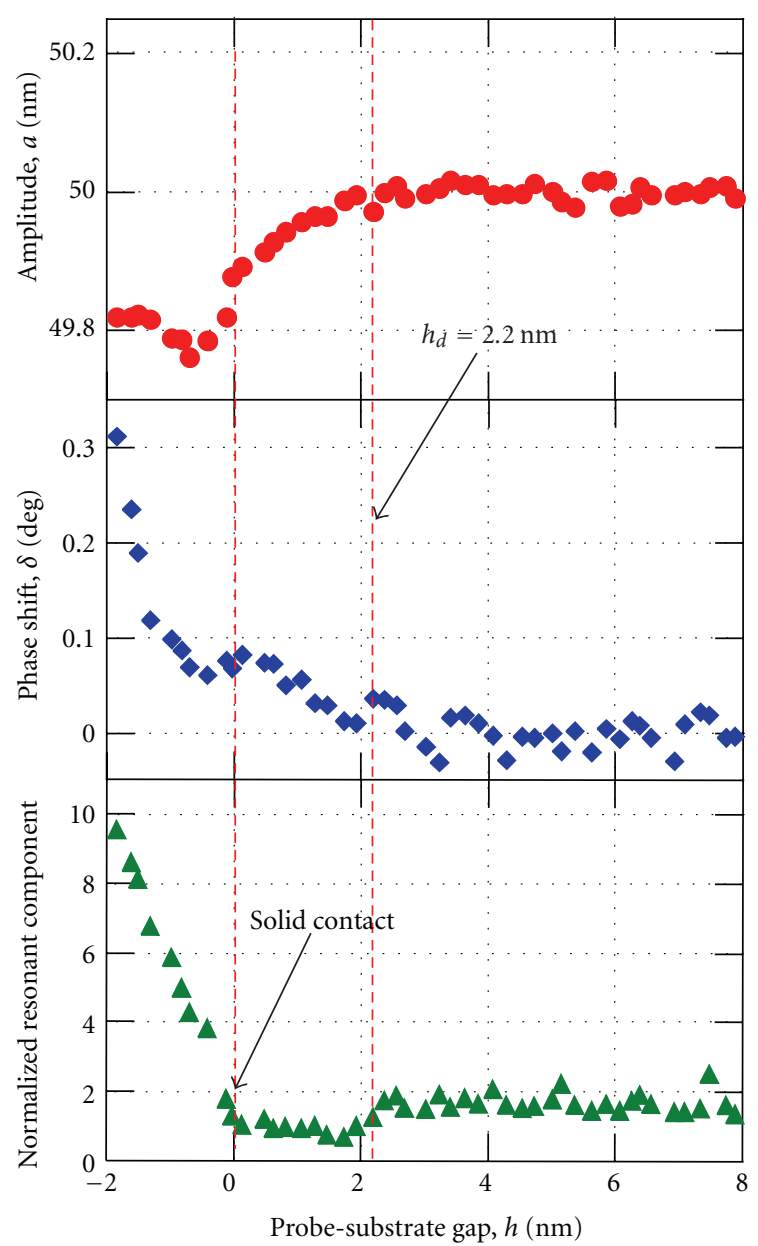

(b) Zdol4000 ( $b=0.2)$

Figure 10: Probe amplitude, phase shift, and normalized resonant component measured for (a) Z03 and (b) Zdol4000 with a bonding ratio of 0.2 in the approaching process [26].

follows the approaching process. In the separating process, the three sequential stages occur in reverse order. These two processes imitate the touchdown and takeoff behavior in the HDI. Although the approaching and separating speeds differ greatly from the actual speeds, we can observe fundamental phenomena. In all measurements, we used an optical fiber probe with a tip radius of curvature of around $8 \mu \mathrm{m}$.

4.2. Calculation of the Viscoelasticity. To calculate $\eta^{\prime}$ and $\eta^{\prime \prime}$ using (9), we need to determine $\Omega$ for this system. Since the gap width will be sufficiently smaller than the radius of curvature of the probe end, we can assume that the geometric configuration of the sliding surfaces is two flat plates moving parallel with a gap of $h$. In that case, $\Omega$ is obtained as follows:

$$
\Omega=\frac{S}{h},
$$

where $S$ represents the contact area between the probe end and lubricant film. It is, however, difficult to determine an accurate value for $S$ experimentally in our setup, which is a major obstacle in obtaining the absolute values of the viscosity and elasticity coefficients from the measured experimental results in the thin-film system. Therefore, we used the relative viscosity $\zeta^{\prime}$ and elasticity $\zeta^{\prime \prime}$ contributions, defined as follows, to discuss the viscoelastic properties [26]:

$$
\begin{gathered}
\zeta^{\prime}=\frac{\eta^{\prime}}{\left(\eta^{\prime 2}+\eta^{\prime 2}\right)}=\frac{a_{0} \sin \delta}{\left(a_{0}^{2}+a^{2}-2 a_{0} a \cos \delta\right)^{1 / 2}}, \\
\zeta^{\prime \prime}=\frac{\eta^{\prime \prime}}{\left(\eta^{\prime 2}+\eta^{\prime \prime 2}\right)^{1 / 2}}=\frac{a_{0} \cos \delta-a}{\left(a_{0}^{2}+a^{2}-2 a_{0} a \cos \delta\right)^{1 / 2}} .
\end{gathered}
$$

For a quantitative comparison, the damping coefficient $c_{f}$ and elastic coefficient $k_{f}$ of the samples can be obtained as follows [28]:

$$
\begin{gathered}
c_{f}=\frac{a_{0}\left(k-m \omega^{2}\right) \sin \delta}{a \omega}, \\
k_{f}=\frac{\left(a_{0} \cos \delta-a\right)\left(k-m \omega^{2}\right)}{a \omega} .
\end{gathered}
$$

\subsection{Results and Discussion}

4.3.1. Touchdown and Takeoff Gap. The touchdown gap width $h_{d}$ was defined by the gap width for which the probe 


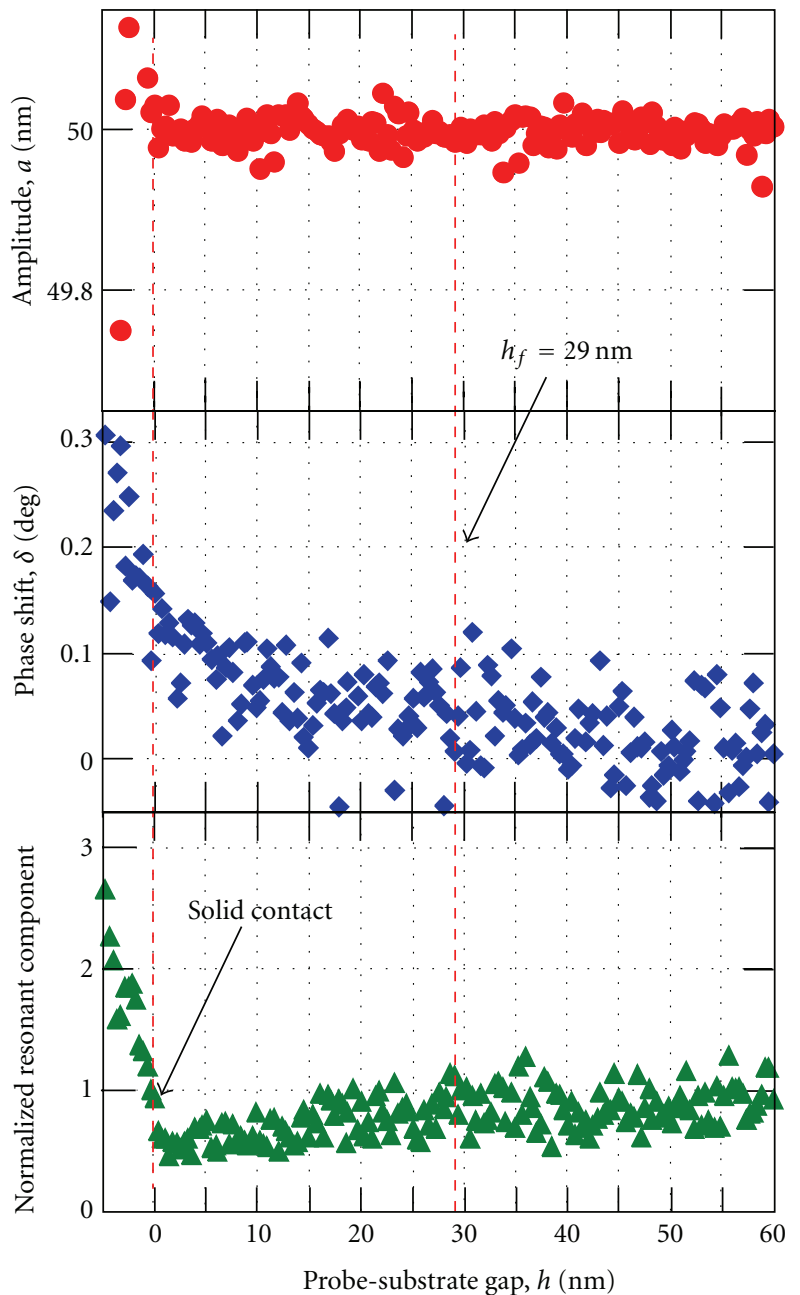

(a) Z03

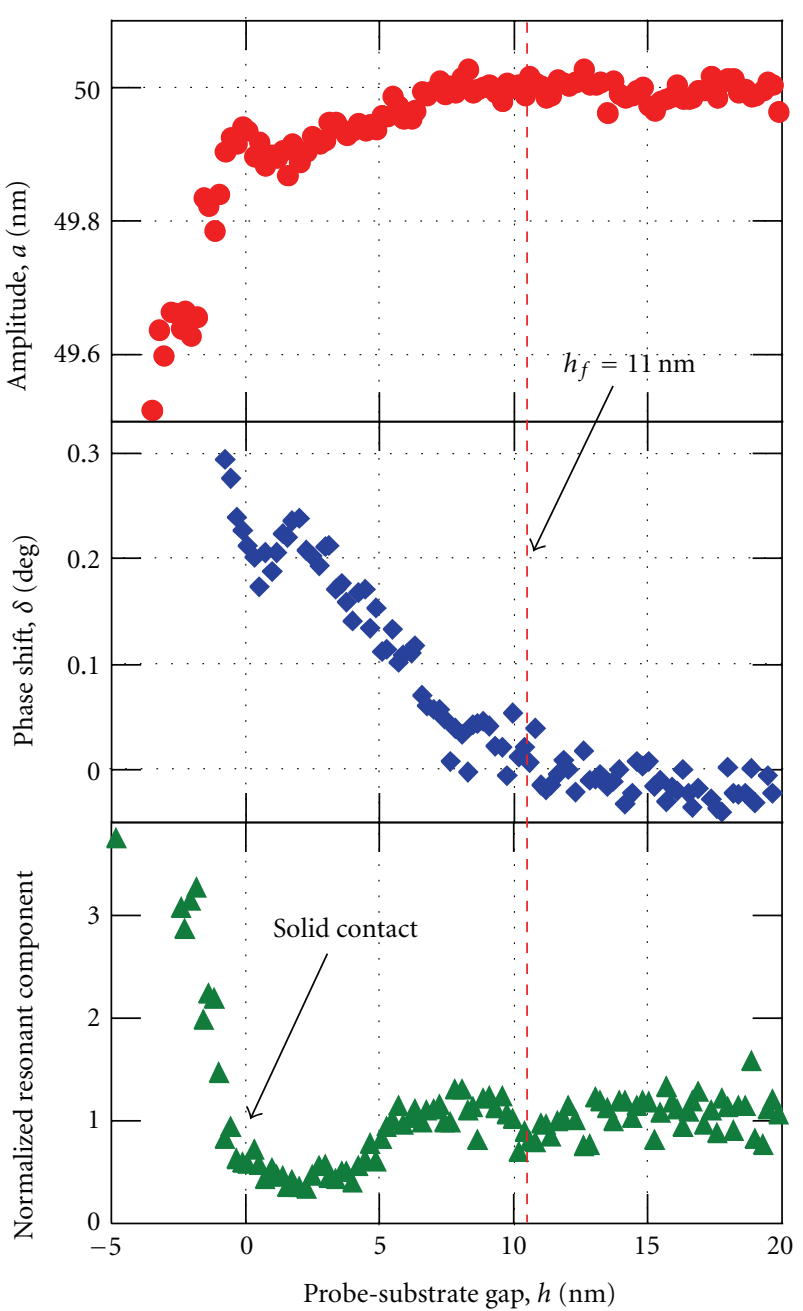

(b) Zdol4000 ( $b=0.2)$

FIGURE 11: Probe amplitude, phase shift, and normalized resonant component measured for (a) Z03 and (b) Zdol4000 with a bonding ratio of 0.2 in the separating process [26].

tip first touches the lubricant film surface in the approaching process. The takeoff gap $h_{f}$ was defined by the gap width for which the probe tip and lubricant film came apart in the separating process. These touchdown and takeoff gaps represent the gap range for which the probe end shears the lubricant film, and they are not necessarily equal to the film thickness since the lubricant film can be deformed. In the approaching process, we determined $h_{d}$ by the gap width for which a decrease in the probe amplitude or an increase in the phase shift was first seen to exceed the noise levels of the measured signal. The $h_{f}$ gap was determined by the gap width for which both an increasing probe amplitude and decreasing phase shift first agreed in the range of noise levels with their average values measured when the probe and the sample were completely apart. The noise levels were obtained by calculating the threefold variance in the measured data for a time when the probe was not in contact with the sample. Typical experimental results from the approaching process are shown in Figures $10(\mathrm{a})$ and 10(b) for Z03 $(b=0)$ and Zdol4000 $(b=0.2)$, respectively. The results measured in the subsequent separating process are shown in Figures 11(a) and 11(b). The touchdown and takeoff gaps are also indicated in Figures 10 and 11. During the approaching process, we observed that a decrease in the probe amplitude and an increase in the phase shift started below a certain gap width. In contrast, the probe amplitude increased and the phase shift decreased during the separating process and reached constant values, which means that the probe end and the sample came apart. The changes in the probe amplitude and phase shift were caused by the viscoelastic force acting on the probe end during the lubricant shearing. The touchdown and takeoff gaps determined for each sample are summarized in Figure 12. The oscillation amplitude was $50 \mathrm{~nm}$ (peak-topeak) and frequency was $1 \mathrm{kHz}$ in all measurements.

While the touchdown gaps of the Zdol4000 and Ztetraol samples had almost the same value as the film thicknesses, that of Z03 was almost twice the film thickness. In the case of $\mathrm{Z} 03$, we consider that the intermolecular attraction from the 


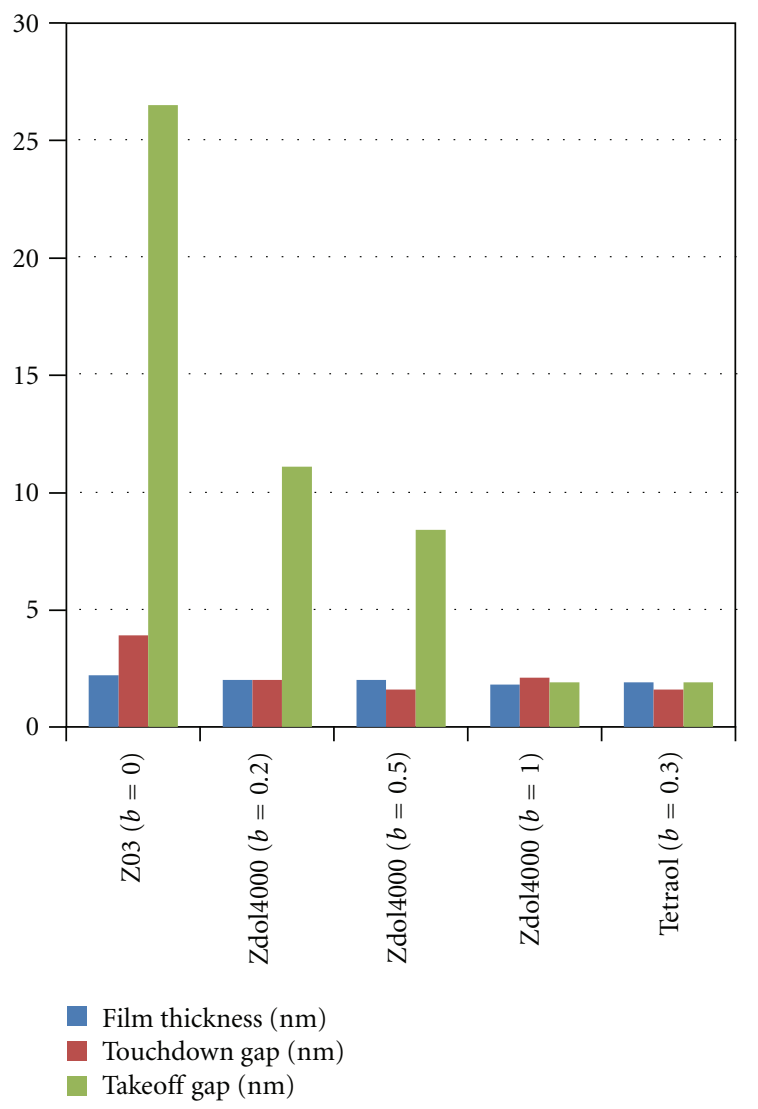

FIGURE 12: Experimental results of the touchdown gaps in the approaching process and takeoff gap in the separating process.

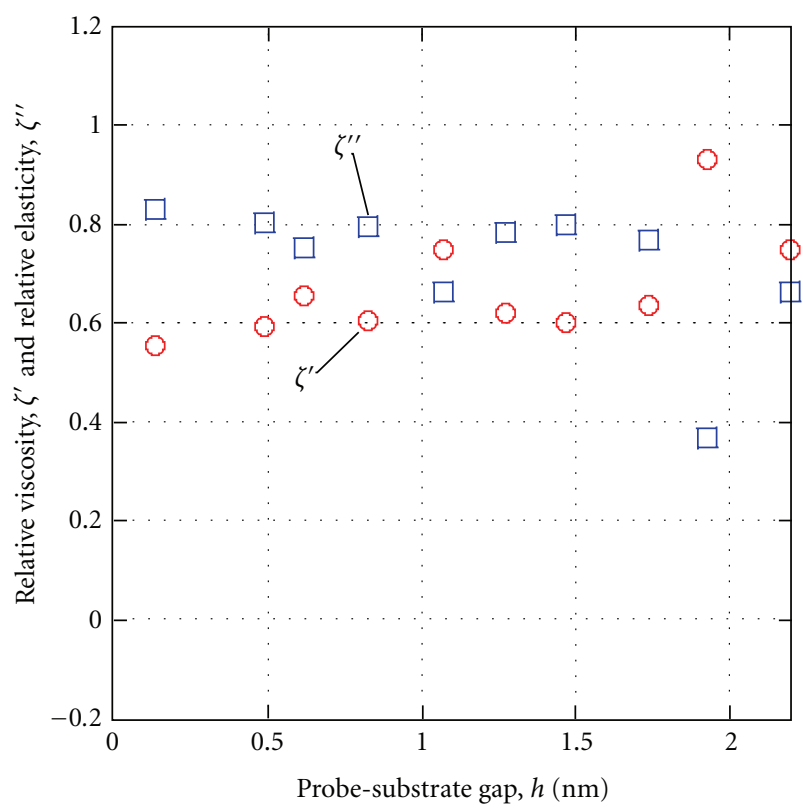

FIGURE 13: Relative viscosity and elasticity of Zdol4000 with a bonding ratio of 0.2 in the approaching process at $h<2.2 \mathrm{~nm} \mathrm{[26].}$

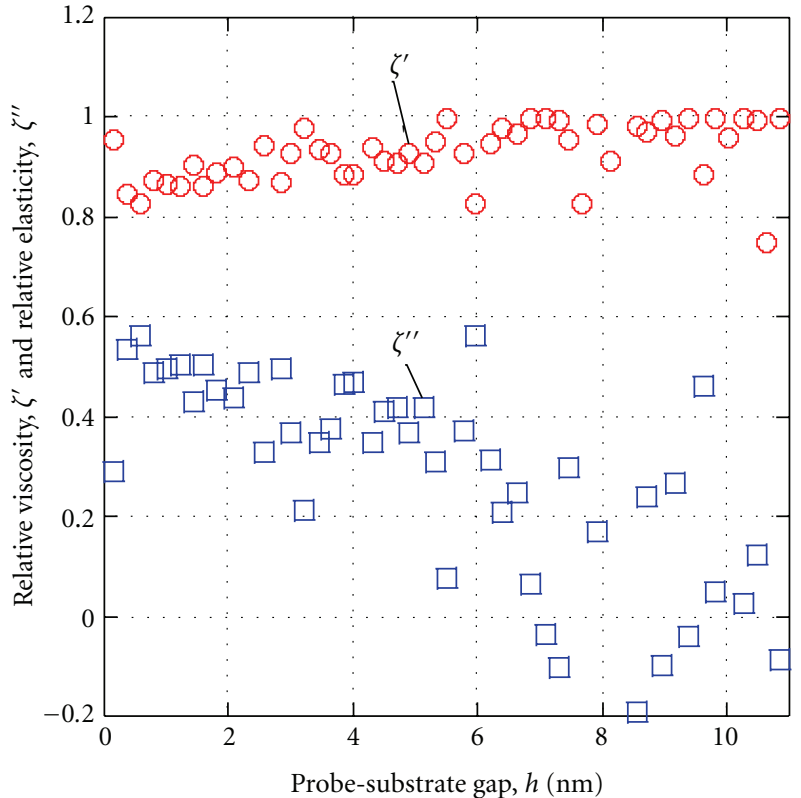

FIGURE 14: Relative viscosity and elasticity of Zdol4000 with a

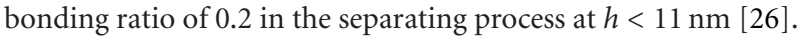

approaching probe tip caused the lubricant film to deform and a liquid bridge to form between the solid surfaces. This results in the contact between the probe tip and lubricant film starting at a gap width larger than the thickness of the lubricant film. A liquid bridge is unlikely to have formed for the Zdol4000 and Ztetraol samples since the touchdown gaps were almost equal to the film thicknesses. We consider these results to be reasonable because the Zdol4000 and Ztetraol molecules could form more energetically stable films than those of Z03 due to the strong interaction of the polar end groups with the disk surface. Since the polar interaction with the disk surface can be much larger than the intermolecular interaction with the probe surface, the Zdol 4000 molecules could not form a liquid bridge.

The takeoff gaps were larger than the film thicknesses except in the case of Zdol4000 $(b=1.0)$ and Ztetraol. In addition, samples with a smaller bonding ratio showed larger takeoff gaps. The small bonding ratio means there is a large number of mobile molecules and therefore suggests that the mobile molecules aggregate near the probe tip during contact with the lubricant film; the increasing gap elongated the cluster to form a liquid bridge that was maintained at gap widths, which were more than a few times larger than the film thickness.

Nonuniformity of the lubricant film thickness can be a significant disturbance to the stable movement of the magnetic head. Our results above indicate that the intermolecular interaction with the approaching probe surface does not cause any deformation in polar lubricant films even if they contain mobile molecules. In contrast, once the probe touches the lubricant film surface the mobile molecules aggregate near the contact region and form a cluster. In future HDDs, frequent contact between the head and disk 


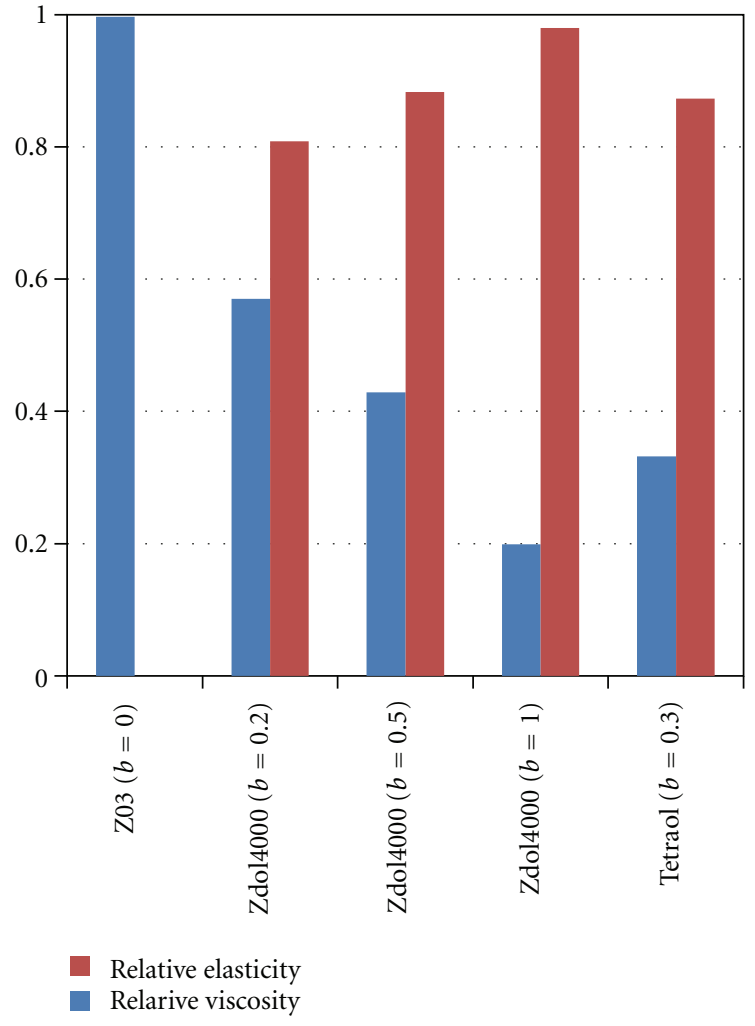

(a) Approaching process

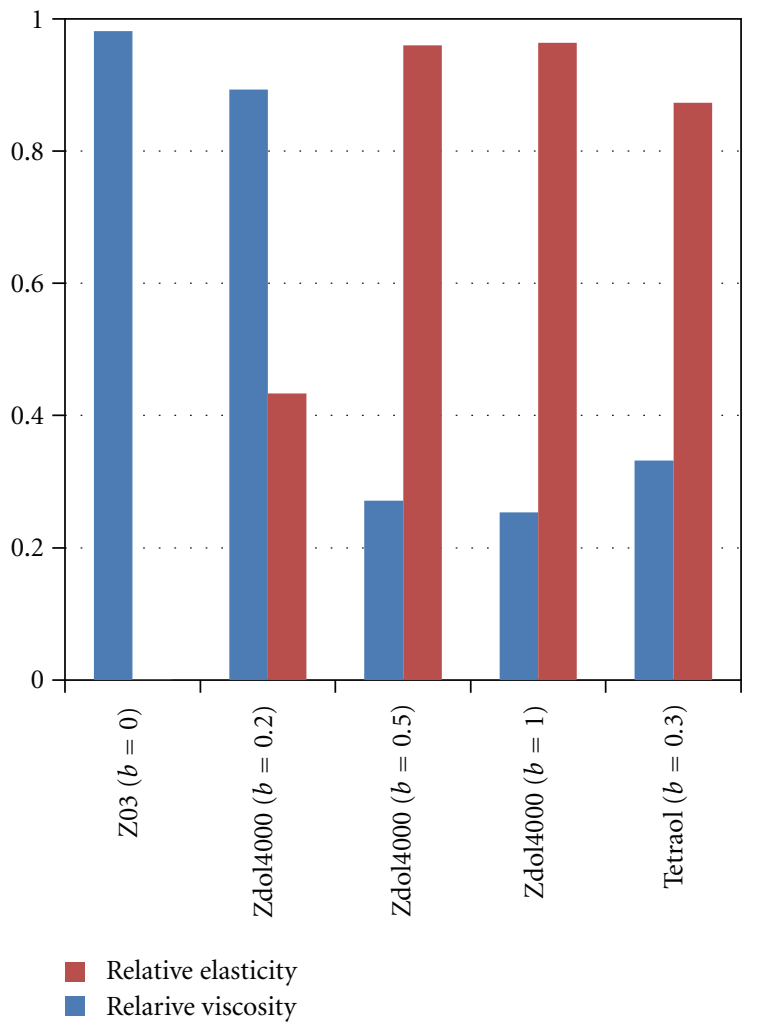

(b) Separating process

FIGURE 15: Relative viscosity and elasticity measured with different samples in (a) approaching and (b) separating processes.

is expected, and thus we suppose that lubricant films with mobile molecules may not be applied for realizing precise control of the magnetic spacing in future HDI. In this case, optimization of the lubricant film design is expected to attain sufficient lubrication without mobile molecules.

4.3.2. Viscoelasticity. We calculated the relative viscosity and elasticity for a gap width less than the touchdown gap in the approaching process and for a gap less than the takeoff gap in the separating process. Typical results for the Zdol400 $(b=0.2)$ sample are shown in Figures 13 and 14, with the circles indicating the relative viscosity and the squares indicating the relative elasticity. The average values in the gap rage from 0 to $1.5 \mathrm{~nm}$ for all the samples are shown in Figure 15. As shown in Figure 15, the behavior of the Z03 $(b=0)$ sample was dominated by the viscosity in both the approaching and separating processes because the Z03 lubricant film is only composed of mobile molecules. The Zdol4000 samples in the approaching process showed a decrease in viscosity and an increase in elasticity with an increase in the bonding ratio. This indicates solidification of the lubricant film caused by an increase in the number of bonded molecules. A significant change in the viscoelastic properties in the approaching and separating processes was observed for the Zdol4000 samples with bonding ratios of 0.2 . While the viscosity was smaller to the elasticity in the approaching process, it became prominent in the separation process. This is caused by the mobile molecules that aggregate around the probe tip. Ztetraol was elasticity dominant in both approaching and separating processes. Although its bonding ratio was 0.3 , the viscoelasticity was qualitatively similar to that of Zdol4000 $(b=1.0)$. Under the sliding condition in this study, the mobile molecules of Ztetraol may behave the same as the bonded ones of Zdol4000. If they are tested under the higher sliding speed, results may be different. This must be one of our future studies. From the results summarized in Figure 15, we can conclude that our method reveals the strong dependence of the viscoelasticity on the lubricant affinity to the disk surface. In addition, the viscoelastic response can be altered locally due to the behavior of mobile molecules.

4.3.3. Shear Thinning Behavior. To reveal the shear rate dependence of the viscoelasticity, we conducted measurements at different oscillating amplitudes of 50, 25, 17.5, 10 , and $5 \mathrm{~nm}$ [28]. The oscillation frequency was fixed at $1 \mathrm{kHz}$ in all of the experiments. The corresponding shear rate ranged from $10^{2}$ to $10^{6} \mathrm{~s}^{-1}$. We used a magnetic disk coated with a $2.0 \mathrm{~nm}$ thick Z03 film. We calculated the damping coefficient $c_{f}$ and elastic coefficient $k_{f}$ from the measured probe amplitude and phase shift by using (13). Since the sample was Z03, which is viscosity dominant, the elasticity $k_{f}$ was quite small and comparable to the noise level. Therefore, at this point, we are not able to discuss the 


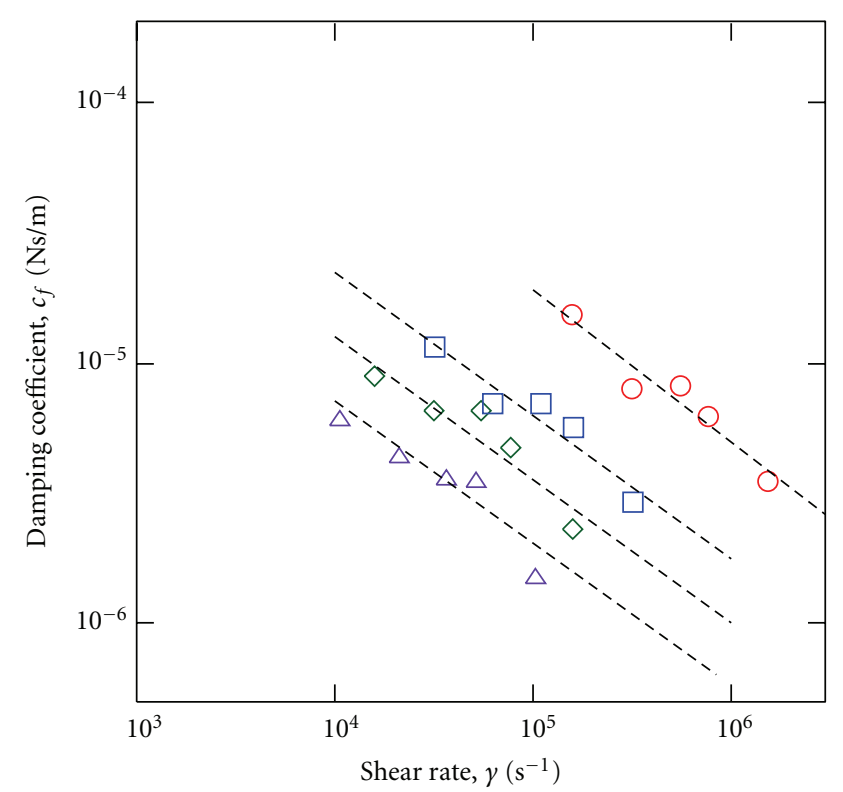

Probe to sample gap: $h$
$h=0.2 \mathrm{~nm}$
$\diamond h=2 \mathrm{~nm}$
$\square h=1 \mathrm{~nm}$
$\triangle h=3 \mathrm{~nm}$

Figure 16: The relationship between the shear rate and viscous coefficient, $c_{f}[28]$.

share rate dependence of $k_{f}$. The shear rate dependence of $c_{f}$ is shown in Figure 16 on a log-log plot. Plots are shown for shearing gaps of $0.2,1.0,2.0$, and $3.0 \mathrm{~nm}$ in the approaching process. The shear rates are calculated from the gap widths and maximum shearing speed, which were determined by the oscillation frequency and amplitude. As shown in Figure 16, $c_{f}$ decreased exponentially with increasing shear rate. This phenomenon is generally known as shear thinning, and the behavior is well expressed by the empirical power law

$$
\log c_{f}=-\alpha \log \gamma+\beta,
$$

where $\alpha$ and $\beta$ are fitting parameters and $\gamma$ represents the shear rate. This is shown by the dashed lines in Figure 16. The shear rate dependence of $c_{f}$ is well represented by the empirical power law. Such a shear rate dependence has not been observed for thicker Z03 films [29], and we therefore consider the shear thinning to be unique to the nanometerthick film.

\section{Conclusion}

We developed the fiber wobbling method (FWM) for dynamic viscoelastic measurements of molecularly thin lubricant films coated on magnetic disk surfaces. The FWM enables us to measure the shear force with a sensitivity of $0.1 \mathrm{nN}$ and control the shearing gap with a resolution on the order of $0.1 \mathrm{~nm}$, both of which are requirements for molecularly thin-film measurements.

We revealed in measurements using the immersed system that lubricants confined in a nanometer-sized gap width had an enhanced viscosity that was several dozen times larger than the bulk viscosity. In addition, the elasticity, which is not observed in the bulk state, appeared at gaps of less than a few nanometers. The appearance of elasticity is considered to be due to the interaction between the adsorbed layers formed on both solid surfaces.

In the thin-film system, we succeeded in measuring the viscosity and elasticity of molecularly thin films and observed a strong dependence of the viscoelasticity on the bonding ratio. In addition, we found that shear thinning caused a decrease in the viscosity as the shear rate increased. Shearthinning viscosity decrease is the opposite effect of the confinement-induced viscosity increase. Therefore, both the shearing gap and shear rate must be considered carefully to predict the friction force acting on the head during contact with the disk surface. The bonding ratio and type of lubricant will also affect the friction force. How these factors define the mechanical properties of the lubricant films must be clarified for the optimal design of future HDI. So far, the FWM has two major drawbacks. One is that the shearing speed is much slower than that in an actual HDI, and the other is that we cannot determine the contact area between the probe tip and lubricant films in the thin-film system, which is the biggest obstacle for a quantitative evaluation of the viscoelasticity. In our recent work, we succeeded in taking high-speed measurements using the resonant oscillation of the probe [30]. Additionally, observation of the contact area in the FWM measurements is one of our ongoing projects, and the first report will appear elsewhere. We believe that the FWM will be a promising and powerful method for improving our understanding of the basic phenomena concerning HDI lubrication.

\section{Acknowledgments}

The present research was partially supported by a Grantin-Aid for Scientific Research from the Japan Society for the Promotion of Science (no. 23686028), by the Storage Research Consortium, and by the Asahi Glass Foundation.

\section{References}

[1] H. W. Hu, G. A. Carson, and S. Granick, "Relaxation time of confined liquids under shear," Physical Review Letters, vol. 66, no. 21, pp. 2758-2761, 1991.

[2] G. Luengo, F. J. Schmitt, R. Hill, and J. Israelachvili, “Thin film rheology and tribology of confined polymer melts: contrasts with bulk properties," Macromolecules, vol. 30, no. 8, pp. 24822494, 1997.

[3] M. Ruths and S. Granick, "Tribology of confined fomblin-Z perfluoropolyalkylethers: molecular weight dependence and comparison between unfunctionalized and telechelic chains," Tribology Letters, vol. 7, no. 4, pp. 161-172, 1999.

[4] G. B. McKenna, "Ten (or more) years of dynamics in confinement: perspectives for 2010," European Physical Journal, vol. 189, no. 1, pp. 285-302, 2010.

[5] G. B. McKenna, "International workshop on dynamics in confinement : a personal summary," Journal de Physique IV France, vol. 10, pp. Pr7-343-PPr7346, 2000. 
[6] G. B. McKenna, "Status of our understanding of dynamics in confinement: perspectives from Confit 2003," European Physical Journal E, vol. 12, no. 1, pp. 191-194, 2003.

[7] G. B. McKenna, "Confit III. Summary and perspectives on dynamics in confinement," European Physical Journal: Special Topics, vol. 141, no. 1, pp. 291-301, 2007.

[8] C. L. Jackson and G. B. McKenna, "The glass transition of organic liquids confined to small pores," Journal of NonCrystalline Solids, vol. 131-133, no. 1, pp. 221-224, 1991.

[9] C. L. Jackson and G. B. McKenna, "Vitrification and crystallization of organic liquids confined to nanoscale pores," Chemistry of Materials, vol. 8, no. 8, pp. 2128-2137, 1996.

[10] W. J. Orts, J. H. Van Zanten, W. L. Wu, and S. K. Satija, "Observation of temperature dependent thicknesses in ultrathin polystyrene films on silicon," Physical Review Letters, vol. 71, no. 6, pp. 867-870, 1993.

[11] J. A. Forrest, K. Dalnoki-Veress, J. R. Stevens, and J. R. Dutcher, "Effect of free surfaces on the glass transition temperature of thin polymer films," Physical Review Letters, vol. 77, no. 10, pp. 2002-2005, 1996.

[12] J. A. Forrest, K. Dalnoki-Veress, and J. R. Dutcher, "Interface and chain confinement effects on the glass transition temperature of thin polymer films," Physical Review E, vol. 56, no. 5, pp. 5705-5716, 1997.

[13] S. Kawana and R. A. L. Jones, "Character of the glass transition in thin supported polymer films," Physical Review E, vol. 63, no. 2, pp. 1-6, 2001.

[14] J. L. Keddie, R. A. L. Jones, and R. A. Cory, "Interface and surface effects on the glass-transition temperature in thin polymer films," Faraday Discussions, vol. 98, pp. 219-230, 1994.

[15] J. Dobbertin, A. Hensel, and C. Schick, "Dielectric spectroscopy and calorimetry in the glass transition region of semicrystalline poly(ethylene terephthalate)," Journal of Thermal Analysis, vol. 47, no. 4, pp. 1027-1040, 1996.

[16] S. Wong, R. A. Vaia, E. P. Giannelis, and D. B. Zax, "Dynamics in a poly(ethylene oxide)-based nanocomposite polymer electrolyte probed by solid state NMR," Solid State Ionics, vol. 86-88, no. 1, pp. 547-557, 1996.

[17] J. Y. Park and G. B. McKenna, "Size and confinement effects on the glass transition behavior of polystyrene/o-terphenyl polymer solutions," Physical Review B, vol. 61, no. 10, pp. 6667-6676, 2000.

[18] F. Kremer, A. Huwe, M. Arndt, P. Behrens, and W. Schwieger, "How many molecules form a liquid?" Journal of Physics Condensed Matter, vol. 11, no. 10, pp. A175-A188, 1999.

[19] K. Tanaka, Y. Tateishi, Y. Okada, T. Nagamura, M. Doi, and H. Morita, "Interfacial mobility of polymers on inorganic solids," Journal of Physical Chemistry B, vol. 113, no. 14, pp. 45714577, 2009.

[20] F. He, L. M. Wang, and R. Richert, "Confined viscous liquids: interfacial versus finite size effects," European Physical Journal: Special Topics, vol. 141, no. 1, pp. 3-9, 2007.

[21] R. D. Priestley, C. J. Ellison, L. J. Broadbelt, and J. M. Torkelson, "Materials Science: structural relaxation of polymer glasses at surfaces, interfaces, and in between," Science, vol. 309, no. 5733, pp. 456-459, 2005.

[22] R. J. Waltman, "Experimental and theoretical investigation of the effect of lubricant structure on the bonding kinetics of perfluoroalkylpolyethers on $\mathrm{CH}(\mathrm{x})$ amorphous hydrogenated carbon," Chemistry of Materials, vol. 12, no. 7, pp. 2039-2049, 2000.

[23] P. H. Kasai, "Carbon overcoat: structure and bonding of ZDOL," Tribology Letters, vol. 13, no. 3, pp. 155-166, 2002.
[24] S. Itoh, K. Fukuzawa, Y. Hamamoto, H. Zhang, and Y. Mitsuya, "Fiber wobbling method for dynamic viscoelastic measurement of liquid lubricant confined in molecularly narrow gaps," Tribology Letters, vol. 30, no. 3, pp. 177-189, 2008.

[25] S. Itoh, K. Fukuzawa, K. Takahashi, T. Ando, H. Zhang, and Y. Mitsuya, "Optimization of dynamic shear force measurement for fiber wobbling method," Microsystem Technologies, vol. 11, no. 8-10, pp. 894-900, 2005.

[26] Y. Hamamoto, S. Itoh, K. Fukuzawa, and H. Zhang, "Viscoelastic properties of monolayer lubricant films during touch-down and take-off behavior measured by fiber wobbling method," Tribology Online, vol. 6, no. 1, pp. 83-95, 2011.

[27] S. Itoh, K. Fukuzawa, Y. Hamamoto, and H. Zhang, "Opposing effects of confinement and confinement-induced shearthinning on viscoelastic properties of liquid lubricant in nanometer-scale gaps," Tribology International, vol. 44, no. 11, pp. 1333-1339, 2011.

[28] Y. Hamamoto, S. Itoh, K. Fukuzawa, and H. Zhang, "Shear thinning behavior of monolayer liquid lubricant films measured by fiber wobbling method," Journal of Physics, vol. 258, no. 1, Article ID 012010, pp. 1-10, 2010.

[29] C. D. Hahm and B. Bhushan, "High shear rate viscosity measurements of perfluoropolyether lubricants for magnetic thinfilm rigid disks," Journal of Applied Physics, vol. 81, no. 8, pp. 5384-5386, 1997.

[30] S. Itoh, K. Fukuzawa, K. Imai, and K. Ishii, "High-Speed Friction Measurements for a Molecularly Thin Lubricant Film Using a Fiber Wobbling Method," in Proceedings of the IEEE International Magnetics Conference (INTERMAG '12), HH-06, Vancouver, Canada, 2012. 

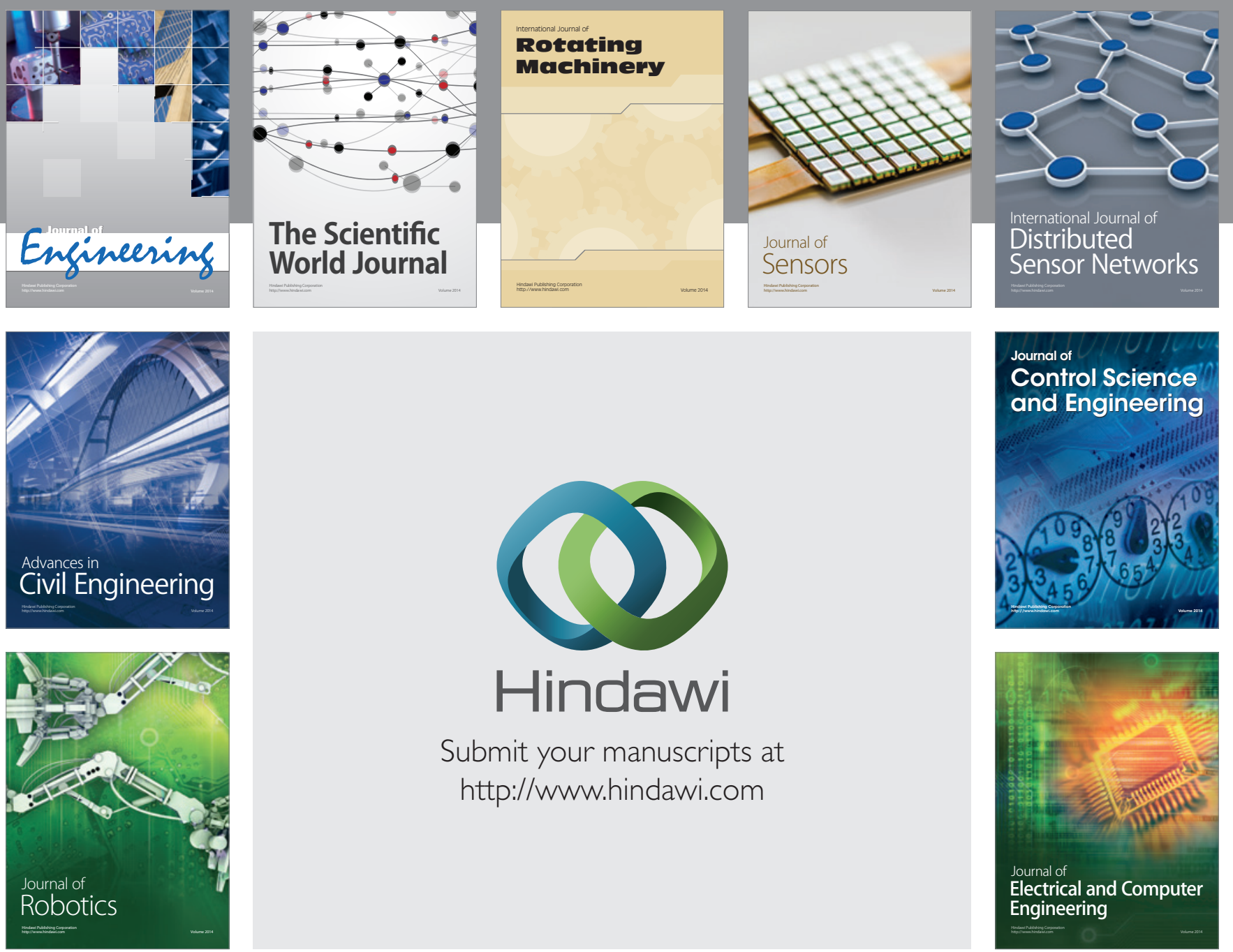

Submit your manuscripts at

http://www.hindawi.com
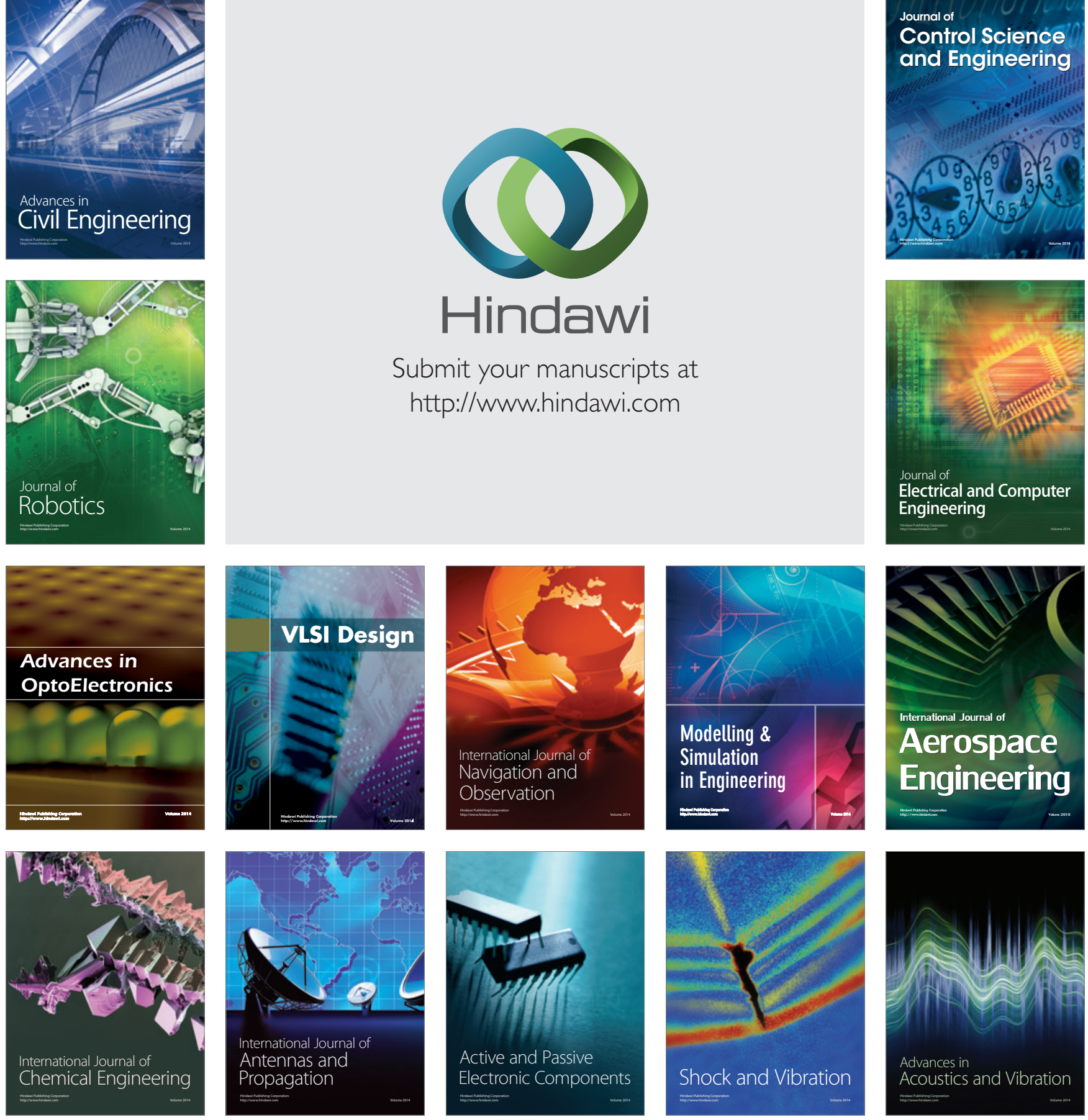